\title{
Interactions and phase behaviour of polyelectrolyte star solutions
}

\author{
Christos N Likos, Norman Hoffmann, Arben Jusufi and Hartmut Löwen \\ Institut für Theoretische Physik II, Heinrich-Heine-Universität Düsseldorf, Universitätsstraße 1, \\ D-40225 Düsseldorf, Germany
}

Received 2 October 2002

Published 16 December 2002

Online at stacks.iop.org/JPhysCM/15/S233

\begin{abstract}
In this work, we first briefly review recent results regarding effective interactions of charged star polymers (polyelectrolyte stars) and then we present new results for many-body systems obtained by employing this interaction. The dominant role is played by the trapped counterions that bring about an entropic repulsion between the centres of these macromolecular aggregates. Subsequently, we explicitly derive density-dependent effective interaction potentials between the polyelectrolyte stars and calculate liquid structure factors and solid-state zerotemperature phase diagrams, discovering thereby a large variety of different crystal phases, similar to those found for the case of neutral star polymers.
\end{abstract}

\section{Introduction}

When polymers containing ionizable groups along their backbone are dissolved into polar solvents, the molecules of the latter pull the aforementioned ionizable groups out of the chains, dissociating them into the solution. In this way, a charged chain, called polyelectrolyte (PE) remains, moving into a solvent that now contains oppositely charged counterions [1]. Attaching now $f$ PEs to a common centre or to the surface of a colloidal particle, of radius $a$, one obtains a PE star [2] or a spherical PE brush [3], respectively. A PE star is the limiting case of a brush when the ratio $a / L$, with $L$ being the brush height, tends to zero. Another example of such a system is provided by the ionizable micelles formed by self-organizing block copolymers. The number of arms $f$ is called functionality of the PE star. The understanding of the properties of PE stars is of great importance for the question of colloidal stabilization, since the grafted brushes act as a barrier against coagulation [2-4]. At the same time, the problem is much more complicated than the related questions of steric and charge stabilization of colloids, because in this case three physical mechanisms are at work: the steric repulsions of the chains, the electrostatic interactions between them, as well as the entropic and screening contributions from the counterions in the solution.

Recently, the conformations of isolated PE stars and their effective interaction were analysed by employing monomer-resolved computer simulations and an analytical theory [5]. 
The physical parameters of the problem, i.e., the functionality $f$, the degree of polymerization $N$ and the fraction of charged monomers $\alpha$, were varied. For charging fractions $1 / 6 \leqslant \alpha \leqslant$ $1 / 2$, it was found that the chains of the stars are strongly stretched, leading to a rod-like configuration. The strong electrostatic attraction between the stars and the counterions leads to an absorption of the majority of the latter inside the star and to partial condensation along the rods. The amounts of both absorbed and condensed counterions depend on the average density of the solution. This result emerges naturally within the variational theory, in which the free energy of the system is written down and minimized with respect to the counterion distribution and the star radius. The theory has been extended to the case of two interacting stars in the solution and the results for the effective force between the star centres are in excellent agreement with simulations [5]. In section 2 we sketch the ingredients of the theoretical approach that allow us to determine the effective star-star interaction at arbitrary density of the solution, and show that this interaction is ultrasoft and becoming softer as the density increases. In section 3 we give a brief account of new results regarding the liquid structure and the crystal phases of PE star solutions and in section 4 we summarize and conclude.

\section{The effective interaction}

We consider PE stars at room temperature, dissolved in water. In this paper, we examine exclusively salt-free solutions. Each charged monomer carries an elementary charge $e$; thus the Bjerrum length $\lambda_{\mathrm{B}}=e^{2} /\left(\epsilon k_{B} T\right)$ has the value 7.1 $\AA$, where $\epsilon=81$ is the dielectric constant of the solvent and $k_{B}$ is Boltzmann's constant. Each chain has $N$ monomers and is charged periodically: every $1 / \alpha$ th monomer along the chain carries charge $e$. Typically $N$ was varied between 50 and 200 and $f$ between 5 and 50. Under these circumstances, the chains assume a stretched configuration; the corona radius $R$ of the stars scales linearly with the degree of polymerization $N$ and is also practically independent of the functionality $f$. The bare charge of the star is $Q_{\text {bare }}=$ ef $N \alpha$; thus there are also $N_{c}=f N \alpha$ monovalent counterions in the solution. The density is $\rho_{\mathrm{s}}=N_{\mathrm{s}} / V$, with $N_{\mathrm{s}}$ the number of stars and $V$ the volume of the system. To deal with the effects of the system's density on the counterion distribution, we introduce a cell model by enclosing the star in a spherical cell of radius $R_{W}=\left(4 \pi \rho_{\mathrm{s}} / 3\right)^{-1 / 3}$. The $N_{c}$ counterions are initially divided into two states: $N_{\text {in }}$ of them are located inside the star and $N_{\text {out }} \equiv N_{3}$ are in the region between $R$ and $R_{W}$ and are called free counterions. These are the charges that screen the Coulomb interaction between the stars at nonoverlapping distances. The $N_{\text {in }}$ trapped counterions can be further divided into two states. $N_{1}$ of them are located at distances $\zeta \leqslant \lambda_{\mathrm{B}}$ from the rods and are thereby condensed around the latter: they execute essentially one-dimensional motions along the stiff chains. The remaining $N_{2}=N_{\text {in }}-N_{1}$ counterions are inside the star but they execute three-dimensional motions, exploring the whole volume inside the star. We call those spherically trapped, in order to distinguish them from the $N_{1}$ condensed ones that are trapped in cylinders of radius $\lambda_{\mathrm{B}}$ along the rods. The three counterion populations are related through $N_{c}=\sum_{i=1}^{3} N_{i}$ and with each state a corresponding entropy is associated [5].

In order to deal theoretically with the effective interaction between two stars whose centres are held at separation $D$, we must take into account two facts. First, for overlapping distances, $D \leqslant 2 R$, no interdigitation between the chains of the two stars takes place. The chains retract as though there were a 'hard plane' perpendicular to the interstar separation vector at its middle. This restriction modifies the spherical charge and monomer distributions of the stars and introduces a peculiar geometry of 'chopped spheres'. The electrostatic and entropic contributions to the free energy can be nevertheless calculated almost analytically even in this case [5]. Second, for nonoverlapping stars, we expect on general grounds based on linear 


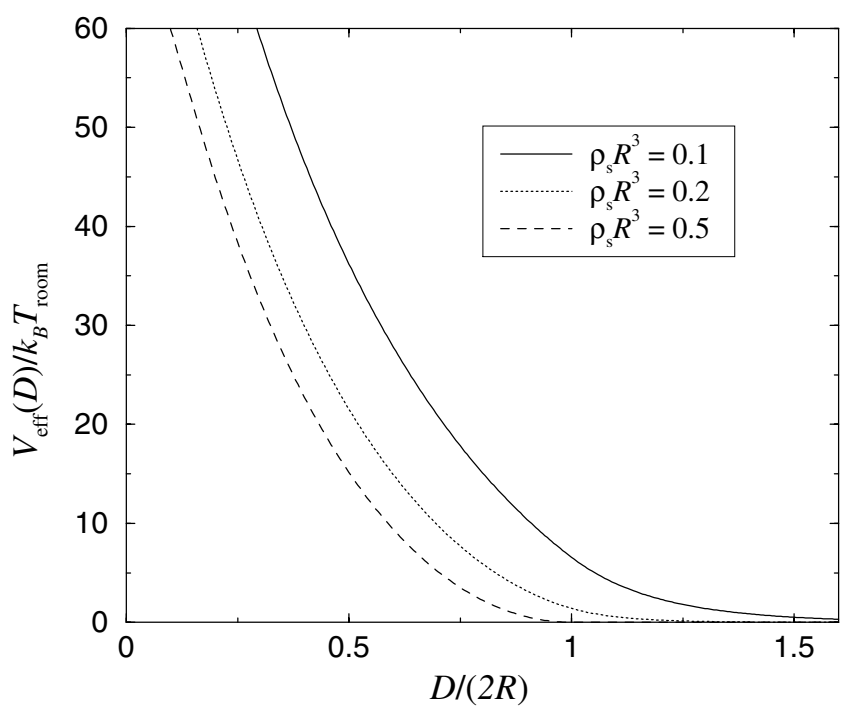

Figure 1. The effective interaction potential for PE stars with $f=10$ and $\alpha=1 / 4$ for three different densities $\rho_{\mathrm{s}}$, as indicated on the plot.

response theory that the effective interaction will be of Yukawa form (screened Coulomb), whereby the screening is caused by the $N_{3}$ free counterions: in this regime, charged stars are identical to charged colloids [6]. Thus, we introduce an effective interaction potential with two branches, $V_{-}(D)$ for $D \leqslant 2 R$ and $V_{+}(D)$ for $D>2 R$, in analogy with the case of neutral stars. The functions $V_{ \pm}(D)$ contain two constants that are then fixed by imposing the physical requirement that both the interaction and the force be continuous at $D=2 R$.

More specifically, the interaction $V_{-}(D)$ is essentially determined by the entropic contributions of the $N_{2}$ spherically trapped counterions. $N_{2}$ is in turn the result of a minimization procedure and is known analytically as a function of $D$. We emphasize that $N_{2}$ depends additionally on $R_{W}$, and hence on the density of the solution; therefore one immediately obtains an explicitly density-dependent potential for overlapping stars. In all results shown below, the number of condensed counterions $N_{1}$ was fixed at the value $N_{1}=0.88 \times N_{1}(2 f)$, where $N_{1}(2 f)$ is the number of condensed counterions of a PE star with $2 f$ arms. This procedure has been shown to yield excellent agreement with simulation for a variety of test cases. We also remark that a change of this parameter has in practice the effect of scaling the whole potential by a constant; hence it causes no changes in the lattice sum phase diagrams to be presented below-it only influences the stability of the fluid phase and hence the location of the freezing phase boundaries. Thereby, the interaction potential for $D \leqslant 2 R$ is obtained in the form $V_{-}(D)=\tilde{V}_{-}(D)+C_{-}$, where $\tilde{V}_{-}(D)$ is a known, analytic function of $D$ (as well as of $R$ and $R_{W}$ ) and $C_{-}$an as yet undetermined constant. The interaction potential for $D>2 R$ has the Yukawa form mentioned above: $V_{+}(D)=C_{+} \exp (-\kappa D) / D$, with a second constant $C_{+}$and the inverse Debye screening length $\kappa$, set by the concentration of the $N_{3}$ free counterions only and given by $\kappa=\sqrt{3 N_{3} \lambda_{\mathrm{B}} /\left(R_{W}^{3}-R^{3}\right)}$. Again, we obtain a densitydependent interaction also for nonoverlapping distances. Here, the density dependence comes both explicitly through $R_{W}$ and implicitly through the dependence of $N_{3}$ on $R_{W}$ and thus on $\rho_{\mathrm{s}}$. Matching the potential energy expressions at $D=2 R$ as described above leads to the full effective interaction potential $V_{\text {eff }}(D)$. 
In figure 1, we show typical results for this effective potential as the density of the solution changes. Note that the overall density dependence of the interaction arises through the variation of $N_{2}$ and $N_{3}$ with $R_{W}$. This can occur only as long as $R_{W}>R$, i.e., below the overlap density $\rho_{\mathrm{s}}^{*}$, defined through the condition $R_{W}=R$ or, equivalently, $\rho_{\mathrm{s}}^{*} R^{3}=3 /(4 \pi) \approx 0.239$. For $\rho_{\mathrm{s}}>\rho_{\mathrm{s}}^{*}$, the interaction vanishes identically for $D>2 R$, as there are no free counterions and the stars, having absorbed all available counterions, are neutral. Hence, they experience no interaction at such separations. The interaction for $D \leqslant 2 R$ tends to zero automatically for $D \rightarrow 2 R^{-}$in this case and also has a vanishing derivative there, as can be seen in figure 1 for the largest displayed density that indeed exceeds the overlap value $\rho_{\mathrm{s}}^{*}$.

\section{Structure and phase behaviour}

Having derived an effective star-star interaction, one can now apply known techniques from liquid- and solid-state theory in order to investigate the pair structure and thermodynamics in the fluid phase, as well as the crystalline phases of the system and phase transitions between them. We start with the latter topic. The pair interaction presented in the preceding section has the characteristic that it is ultrasoft. With this term, we mean that the repulsive interaction potential grows as $D$ approaches zero much more slowly than any power law; as a matter of fact, for model PE stars with point-like centres, this potential is even bounded: $V_{\text {eff }}(D=0)<\infty$. Such ultrasoft interactions have attracted quite some attention recently, due to the fact that they are quite common in soft-matter systems: polymer chains [7], neutral star polymers [8], as well as dendrimers [9] are all typical 'polymeric colloids' that interact by means of effective potentials belonging to this class, and spherical PE brushes are also members of this family, provided that the ratio of brush height to core radius is high.

As far as crystal phases of ultrasoft colloids are concerned, it has been recently demonstrated that these systems defy the conventional wisdom gained from atomic, hardsphere-like fluids. Guided by that paradigm, one expects that

(i) the high-density state of classical fluids is crystalline and

(ii) the fcc and bec crystals are natural candidates for constituting the stable solids.

Ultrasoft colloids show different behaviour, ranging from complete lack of crystallization to re-entrant melting upon density increase [10-12] to the existence of different crystal structures above the overlap density $[10,13]$. In order to examine the persistence of such freezing patterns also for the interaction potential at hand, we have used it as input in 'zero-temperature' lattice sum calculations of the ground-state energy for a variety of candidate crystals.

We examined the following structure groups: the body-centred orthogonal (bco) structures that include the bcc and fcc lattices as special cases; the tetragonal lattices that include the simple cubic (sc) lattice as a special case; and two non-Bravais lattices-the diamond structure as well as the A15 structure [14]. The results of the lattice sum calculations for a fixed charge ratio $\alpha=1 / 3$ and varying functionality $f$ are shown in figure 2 . We obtain a rich variety of solid phases, whose succession is very similar to that obtained for neutral star polymers $[10,13]$. However, in the case at hand, the bcc and sc crystals that were not stable for neutral stars do appear in the lattice sum phase diagram. Moreover, at higher densities, $\rho_{\mathrm{s}} R^{3} \gtrsim 1$, the A15 phase is stable among all crystal structures considered but it does not appear yet in the phase diagram since the fluid is more stable there (see below). However, we anticipate that at high functionalities $f$, for which the interaction potential becomes stronger, the A15 phase will regain thermodynamic stability from the fluid and will thus materialize. This finding is in agreement with the arguments put forward in [14]. All transitions between crystals are first order with very small density gaps. 


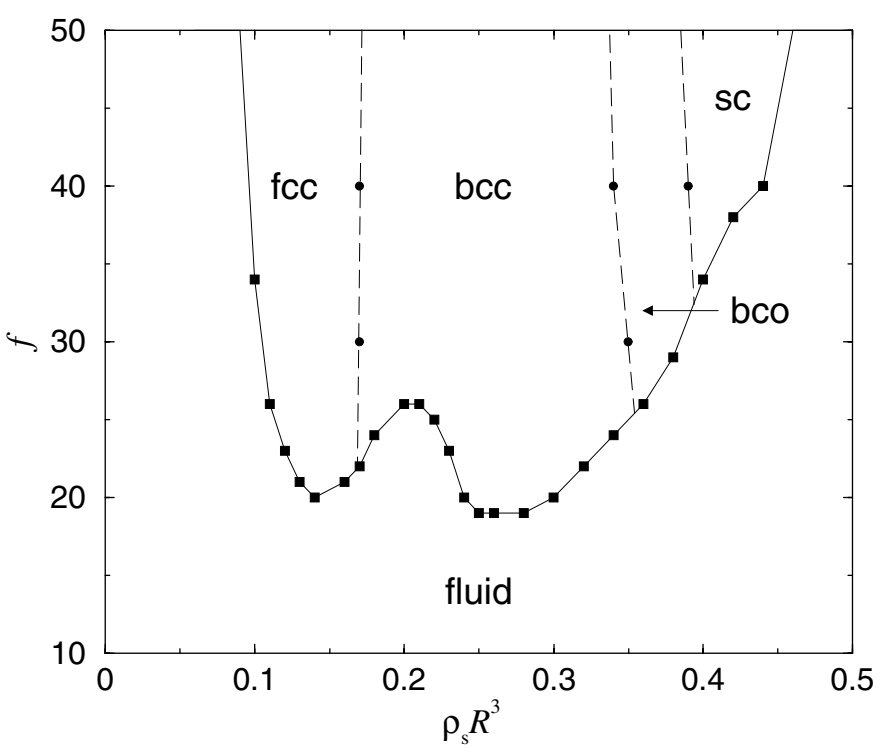

Figure 2. The phase diagram of PE stars with fixed charge ratio $\alpha=1 / 3$, drawn in the density functionality plane. The points indicating solid-solid coexistence were calculated using lattice sums for functionality values $f=10,20,30,40$ and 50 and the dashed lines connecting them are a guide to the eye. Points along the fluid-freezing boundary (connected by the solid line) were obtained via the Hansen-Verlet freezing rule.

To estimate the stability of the fluid phase, we have calculated liquid structure factors $S(q)$ within the hypernetted chain closure. The freezing line has been obtained using the Hansen-Verlet rule by fixing the amplitude of the first peak in $S(q)$ to 2.85 . The freezing density behaves nonmonotonically with the arm number $f$. In fact, for fixed arm numbers in the range of $20 \lesssim f \lesssim 25$, a sequence of two re-entrant melting transitions occur. This is also reflected in anomalous liquid structure factors, similarly to the case for neutral stars [16]. The detailed calculation of the phase diagram by means of evaluation of the free energy of the various phases is a demanding task that is further complicated by the density dependence of the interaction; results along these lines will be presented elsewhere [17]. Nevertheless, the combination of lattice sum calculations for the crystals and the Hansen-Verlet criterion for the fluid has been proven to be a very reliable method $[10,11]$; thus we anticipate that the phase diagram shown here already captures the features of the thermodynamic behaviour of PE star solutions.

\section{Conclusions}

The effective interaction between star-branched PEs has been derived and it has been shown that it is genuinely density dependent. As a general characteristic, this interaction is ultrasoft, a fact that has the consequence of bringing about different stable crystal phases as well as a double re-entrant melting. These characteristics are akin to those observed for neutral star polymers. However, in the case at hand, the physical origin of the interaction lies in the entropy of the trapped counterions and not in the self-avoidance of the chains or the electrostatics. Moreover, the charge ratio and the salt concentration provide two more tunable physical parameters that can lead to a very rich structural and phase behaviour. 


\section{References}

[1] See, e.g., Joanny J F 2001 Eur. Phys. J. E 53 and other articles contained in this special issue on polyelectrolytes

[2] Pincus P 1991 Macromolecules 242912

[3] Guo X and Ballauff M 2000 Langmuir 168719

[4] Groenenwegen W, Lapp A, Egelhaaf S U and van der Maarel J R C 2000 Macromolecules 334080

[5] Jusufi A, Likos C N and Löwen H 2002 Phys. Rev. Lett. 88018301 Jusufi A, Likos C N and Löwen H 2002 J. Chem. Phys. 11611011

[6] Denton A R 2003 Phys. Rev. E at press

[7] Louis A A, Bolhuis P G, Hansen J-P and Meijer E J 2000 Phys. Rev. Lett. 852522 Louis A A, Bolhuis P G and Hansen J-P 2000 Phys. Rev. E 627961

[8] Likos C N, Löwen H, Watzlawek M, Abbas B, Jucknischke O, Allgaier J and Richter D 1998 Phys. Rev. Lett. 804450

[9] Likos C N, Schmidt M, Löwen H, Ballauff M, Pötschke D and Lindner P 2001 Macromolecules 342914 Likos C N, Rosenfeldt S, Dingenouts N, Ballauff M, Lindner P, Werner N and Vögtle F 2002 J. Chem. Phys. 1171869

[10] Watzlawek M, Likos C N and Löwen H 1999 Phys. Rev. Lett. 825289

[11] Lang A, Likos C N, Watzlawek M and Löwen H 2000 J. Phys.: Condens. Matter 125087

[12] Likos C N, Lang A, Watzlawek M and Löwen H 2001 Phys. Rev. E 63031206

[13] Likos C N, Hoffmann N, Löwen H and Louis A A 2002 J. Phys.: Condens. Matter 147681

[14] Ziherl P and Kamien R 2000 Phys. Rev. Lett. 853528 Ziherl P and Kamien R 2001 J. Phys. Chem. B 10510147

[15] Balagurusamy V S K, Ungar G, Percec V and Johansson G 1997 J. Am. Chem. Soc. 1191539

[16] Watzlawek M, Likos C N and Löwen H 1998 J. Phys.: Condens. Matter 108189

[17] Hoffmann N, Likos $\mathrm{C} N$ and Löwen $\mathrm{H}$ in preparation 